\title{
The Efficacy of Infliximab in Sciatica Induced by Disc Herniations Located at L3/4 or L4/5: A Small-Scale Randomized Controlled Trial
}

\author{
Jaro Karppinen ${ }^{*}, 1,6$, Timo Korhonen ${ }^{1}$, Anthony Hammond ${ }^{2}$, Chris Bowman ${ }^{3}$, Antti Malmivaara ${ }^{4,6}$, \\ Nic Veeger ${ }^{5}$, Seppo Seitsalo ${ }^{6}$ and Heikki Hurri ${ }^{6}$ \\ ${ }^{I}$ Department of Physical and Rehabilitation Medicine, University of Oulu, Oulu, Finland \\ ${ }^{2}$ Maidstone Hospital, Kent, UK \\ ${ }^{3}$ Tonbridge Clinic, 339 Shipbourne Road, Tonbridge, Kent, UK \\ ${ }^{4}$ Finnish Office for Health Technology Assessment, Helsinki, Finland \\ ${ }^{5}$ Trial Coordination Center, University Medical Center Groningen, University of Groningen, Groningen, The \\ Netherlands \\ ${ }^{6}$ ORTON Orthopaedic Hospital of Invalid Foundation, Helsinki, Finland
}

\begin{abstract}
Objective: To evaluate the efficacy of Infliximab, a monoclonal antibody against tumor necrosis factor alpha (TNF $\alpha$ ), in patients with acute/subacute sciatica secondary to herniated disc at L3/4 or L4/5.

Design: A randomized controlled trial.

Background: The results from our randomized trial demonstrated no efficacy for Infliximab but the subgroup results suggested that Infliximab may be effective for sciatica induced by herniations at L3/4 or L4/5.

Methods: Inclusion criteria were unilateral moderate-to-severe sciatic pain with an MRI-confirmed disc herniation at L3/4 or L4/5 and candidacy for discectomy. Patients were randomized to receive either a single infusion of Infliximab $5 \mathrm{mg} / \mathrm{kg}$ or placebo. Outcomes included intensity of leg and back pain, Oswestry disability, quality-of-life (RAND-36) and straight leg raising (SLR) restriction at week 26. Data between baseline and the six-month follow-up were analyzed using MannWhitney U test.

Results: Due to slow recruitment the trial ended prematurely after 15 patients in total were allocated to receive Infliximab $(n=7)$ or placebo $(n=8)$. At week 26 , leg pain intensity had decreased by $73 \%$ in the Infliximab group compared to $65 \%$ in the control group $(\mathrm{p}=0.52)$. For all measured variables at 6 months, Infliximab treatment was associated with greater improvements compared to placebo, especially at early time points. Two patients in the Infliximab group and three in the placebo group had a discectomy or caudal injection $(\mathrm{p}=1.00)$.
\end{abstract}

Conclusions: Our results warrant continuation of research on TNF $\alpha$ antagonists in sciatica induced by disc herniations at $\mathrm{L} 3 / 4$ or L4/5.

\section{INTRODUCTION}

Tumor necrosis factor alpha (TNF $\alpha)$ has been established as an important mediator in intervertebral disc herniationinduced sciatica in animal models $[1,2]$. The results of our uncontrolled, open-label study in 10 subjects showed a beneficial effect of a single infusion of Infliximab, a monoclonal antibody against $\mathrm{TNF} \alpha$, for herniation-induced sciatica in significantly reducing leg pain and back-related disability $[3,4]$. However, the results from our randomized controlled trial in 40 patients with disc herniation-induced sciatica demonstrated no difference in efficacy between Infliximab and placebo in reducing leg pain from baseline to 12 weeks [5].

*Address correspondence to this author at the Department of Physical and Rehabilitation Medicine, University of Oulu, Box 25, 90029 University of Oulu, Finland; Fax: +358-8-3152398; E-mail: jaro.karppinen@ttl.fi
Interestingly, subgroup analysis of the one-year followup of FIRST 2 indicated that Infliximab may be more effective in treating sciatica induced by disc herniations at L3/4 or L4/5 [6]. Therefore, the aim of the current study was to evaluate the efficacy of Infliximab $5 \mathrm{mg} / \mathrm{kg}$ for sciatica due to disc herniations at $\mathrm{L} 3 / 4$ or $\mathrm{L} 4 / 5$ in a randomized controlled trial.

\section{PATIENTS AND METHODS}

\section{Patients}

Patients were recruited at two Finnish centers (Oulu University Hospital, Oulu and ORTON Orthopedic Hospital, Helsinki) and one center in the UK (Maidstone Hospital, Kent). Centocor Inc (Horsham, PA, USA) funded the trial and provided the study agent. The Oulu University Hospital Ethics Committee (Oulu, Finland) and the Maidstone and Tunbridge Wells Local Ethics Committee (Kent, UK) 
approved the study protocol. All patients provided written informed consent.

Eligible patients had unilateral sciatic pain in conjunction with a magnetic resonance imaging (MRI)-confirmed disc herniation at L3/4 or L4/5 concordant with the symptoms and signs of radicular pain. Patients also had to be candidates for discectomy, as determined by an independent orthopedic surgeon. In addition to a disc herniation on MRI concordant with related clinical signs and symptoms of radicular pain, criteria for discectomy included neural entrapment (straight leg raising $(\mathrm{SLR}) \leq 60^{\circ}$ ) with 4 to 12 weeks of moderate-tosevere leg pain. Patients had to be older than 18 years and receiving nonsteroidal anti-inflammatory drugs (NSAIDs) at the recommended minimum dose for at least 2 weeks prior to study entry. Exclusion criteria included e.g. need for emergency surgery and history of back surgery [5].

\section{Study Design and Clinical Evaluations}

Patients were screened for tuberculosis [5]. The clinical examination included a review of medical history as well as disease-specific parameters including straight leg raise (SLR), lumbar flexion, side bending, tendon reflexes, manual motor, and sensory testing. Patient-recorded subjective symptom assessments included a $100-\mathrm{mm}$ visual analog scale (VAS) for leg and back pain, the Oswestry back-related disability index, the number of sick leave days due to the current sciatic episode, and quality-of-life assessed by the RAND-36.

Patients were allocated to treatment groups using random number tables at each center. Patients received a single intravenous infusion of either Infliximab $5 \mathrm{mg} / \mathrm{kg}$ or placebo (saline) over a 2-hour period; vital signs were assessed throughout the infusion. Leg and back pain were assessed 3 hours after the initiation of the infusion and by phone on Day 1 after the infusion. The clinical examination was repeated after the cessation of the infusion. Follow-up assessments (including clinical examinations and subjective symptom assessments) were performed at 1 and 2 weeks; and 1, 3 and 6 six months after the infusion. The occurrence of adverse events and serious adverse events and the number of discectomies were monitored and recorded.

\section{Statistical Analysis}

Twenty patients per group were needed to achieve $80 \%$ power to detect a difference in reduction of leg pain at 12 weeks between the Infliximab (estimated $45 \mathrm{~mm}$ reduction) and the placebo (estimated $20 \mathrm{~mm}$ reduction) groups at a significance level of $5 \%$ and assuming a standard deviation of $30 \mathrm{~mm}$ for both.

In the primary evaluation, Infliximab-treated patients were compared with placebo-treated patients with regard to improvement of the assessed outcomes through the 6-month period following the single infusion. The primary study outcome was the effect of Infliximab versus placebo on the reduction of leg pain (VAS). Other outcomes were back pain (VAS), the number of discectomies, reduction of SLR restriction, reduction of back-related disability (Oswestry), change in health-related quality-of-life (RAND-36), and the number of days on sick leave.
Treatment group differences between continuous variables were compared with the Student or Mann-Whitney $\mathrm{U}$ test, depending on the normality of data (assessed by Shapiro-Wilk test). A Fisher's exact test or Chi-square test was employed for categorical variables. The last-valuecarried-forward method was applied for patients with treatment failure, i.e., surgery or other invasive procedure required (selective nerve root block or epidural injection) during the 6-month period, or patients missing data at the 26week assessment. In addition, leg and back pain, Oswestry disability, and SLR restriction were analyzed using repeated measures analysis with a general linear mixed model with fixed times and covariates, including the baseline value of the variable of interest, duration of current sciatic episode, and age.

Statistical significance was based on a two-sided p-value of $<0.05$. All analyses were performed on an intention-totreat basis, using $\mathrm{SAS}^{\circledR}$ for Windows ${ }^{\mathrm{TM}}$ version 9.1 (Cary, N.C.) or SPSS 11.5.1 (SPSS Corporation, Chicago, IL) for logistic regression analyses.

\section{RESULTS}

\section{Baseline Characteristics and Disposition}

From November 2003 through November 2006, 15 patients were randomized to receive Infliximab $5 \mathrm{mg} / \mathrm{kg}(\mathrm{n}=7)$ or placebo $(n=8)$ before the trial was prematurely terminated due to slow recruitment across all three centers. The mean age of the patients was 53 years, while $67 \%$ of them were male. The mean duration of symptoms was 58 days (range 23 to 91 days), leg pain intensity at baseline $69 \mathrm{~mm}$, Oswestry disability score $62 \%$, and SLR 35 degrees. Thirteen herniations were located at $\mathrm{L} 4 / 5$ and two at L3/4. No significant differences in baseline variables were observed between groups. Two patients dropped out at week 1: one patient allocated to Infliximab from the Helsinki center withdrew the consent, one patient allocated to placebo withdrew for unknown reasons. One patient allocated to placebo from the Kent center missed the six-month visit. Therefore, these patients were excluded from the efficacy analyses. Last-value-carried-forward was used in case of treatment failures. In additional analyses it was also used for patients with missing data $(n=3)$ but did not change the results significantly.

\section{Leg Pain}

At week 12, the absolute median change in leg pain as assessed by VAS was $-52 \mathrm{~mm}$ (range -2 to $-82 \mathrm{~mm}$ ) in the Infliximab group compared with $-34 \mathrm{~mm}(5$ to $-63 \mathrm{~mm})$ in the placebo group $(\mathrm{p}=0.15)$. The absolute median change in leg pain at week 26 was $-47 \mathrm{~mm}$ (range -2 to $-84 \mathrm{~mm}$ ) in the Infliximab group compared with $-45 \mathrm{~mm}(5$ to $-62 \mathrm{~mm})$ in the placebo group (Table 1). At six months, leg pain intensity decreased from baseline by $73 \%$ in the Infliximab group compared with $65 \%$ in the placebo group $(\mathrm{p}=0.52$; Fig. 1a).

\section{Back Pain, Back-Related Disability and Health-Related Quality-of-Life}

At week 12, the absolute median change in back pain was $-41 \mathrm{~mm}$ (range 26 to $-76 \mathrm{~mm}$ ) in the Infliximab group compared with $-7 \mathrm{~mm}$ ( 25 to $-41 \mathrm{~mm})$ in the placebo group 

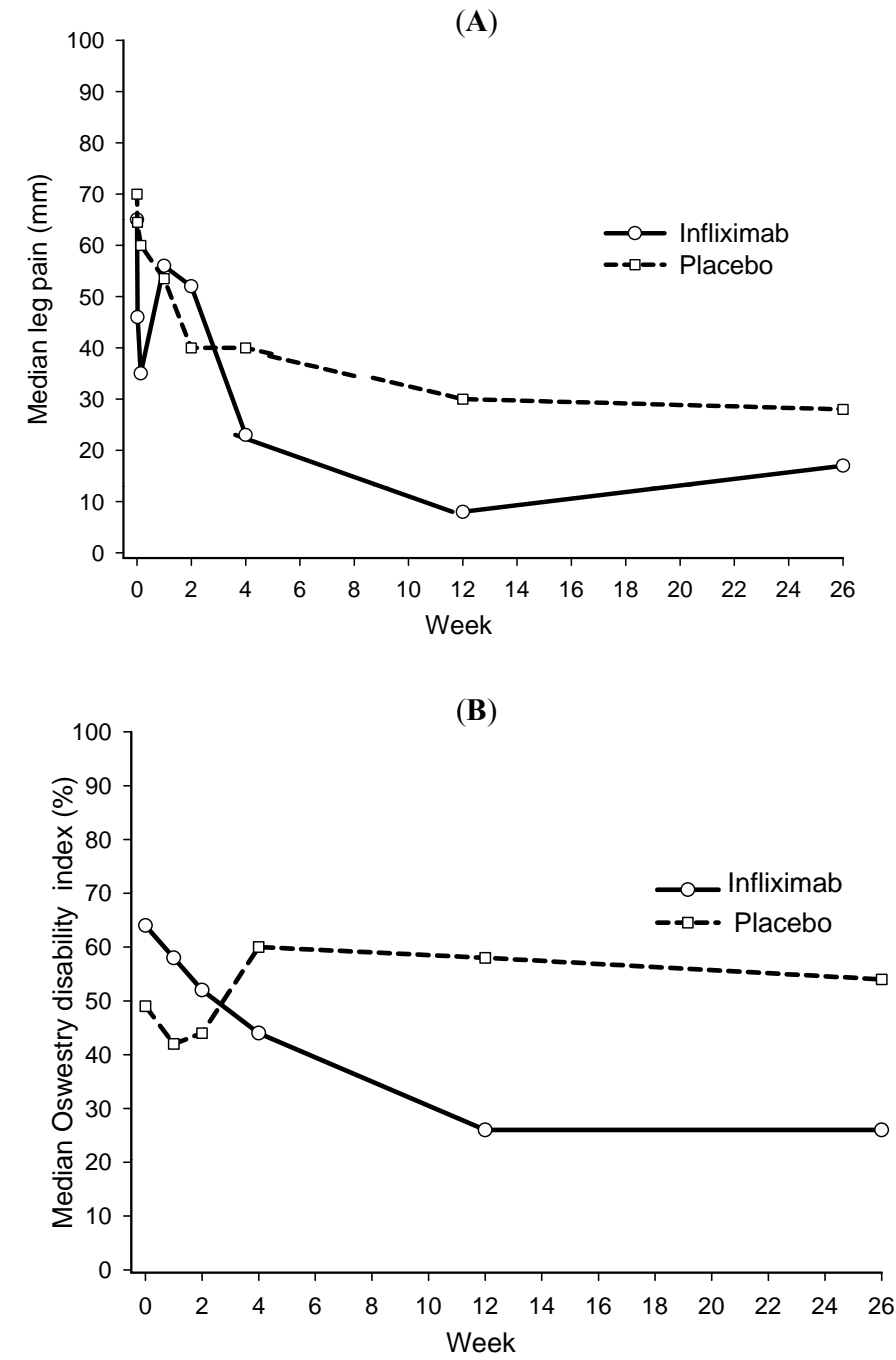

Fig. (1). (A) Median intensity of leg pain on a 100-mm Visual Analog Scale and (B) median Oswestry score (\%) after a single infusion of study agent.

$(p=0.13)$. At week 26, the absolute median change in back pain was $-27 \mathrm{~mm}$ (range 26 to $-78 \mathrm{~mm}$ ) in the Infliximab group compared with $-14 \mathrm{~mm}(3$ to $-71 \mathrm{~mm})$ in the placebo group $(\mathrm{p}=0.25$; Table 1$)$.

The absolute median change in the Oswestry score was 19 (range 2 to -30 ) in the Infliximab group compared with $1(2$ to -15$)$ in the placebo group $(\mathrm{p}=0.57$; Table 1$)$. At week 26 , Oswestry disability score had decreased from baseline by $59 \%$ in the Infliximab group compared with $3 \%$ in the placebo group (Fig. 1b).

At week 26, a trend towards superiority of Infliximab was observed in RAND-36 physical functioning and general health subscales (Table 1).

\section{Clinical Examination Findings, Treatment Failures, and Adverse Events}

The median change in SLR was $35^{\circ}$ (range -0 to $60^{\circ}$ ) in the Infliximab group, compared with $22^{\circ}$ (range 10 to $60^{\circ}$ ) in the placebo group $(p=0.74)$. No significant differences were observed between the groups in the normalization of motor strengths or sensory defects (data not shown).
At week 26, 2 of 7 patients had discectomies or caudal epidural in the Infliximab group compared with 3 of 8 patients in the placebo group $(\mathrm{p}=1.00)$.

Adverse events had occurred in 1 of 7 patients in the Infliximab group (basal cell carcinoma removed from the neck) compared with 2 of 8 patients in the placebo group (Meniere's disease and headache; osteoarthritis of thumb) $(p=1.00)$. No serious adverse events were observed during the infusion period or throughout the six-month follow-up.

\section{DISCUSSION}

Treatment with Infliximab, an anti-TNF $\alpha$ treatment, in sciatica induced by disc herniations at L3/4 or L4/5 resulted in greater improvement in all measured variables, particularly at early time points, but these changes did not reach statistical significance.

Although the results of the first randomized trial performed on the efficacy of anti-TNF $\alpha$ treatment in sciatica, were not positive [5], we observed that Infliximab tended to be more effective in patients with sciatica induced by disc herniations at L3/4 or L4/5 [6]. This led to the current randomized trial, which was performed in one center in the UK and two in Finland. Patients had to be candidates for discectomy with disc herniations at L3/4 or L4/5. Unfortunately, only 15 out of 40 intended patients were recruited, which led to discontinuation of the trial.

Currently, a body of clinical evidence suggests that conservative treatments have not been successful in treating sciatica [7] and that while surgery has demonstrated treatment benefit in herniation-induced sciatica, the outcomes from surgical and conservative strategies have been shown to approach each other within 2-10 years [8-11]. Regardless, effective conservative treatment options are needed as surgical procedures are associated with greater complications. Furthermore, MRI follow-up examinations indicate that herniations tend to regress over time, with partial to complete resolution within three to six months in the majority of subjects [12-14].

The results of our trial are encouraging, e.g., in the case of the Oswestry score, a $50 \%$ reduction in disability was observed in the Infliximab group compared to virtually no change in the placebo group. Similar treatment responses in favour of Infliximab were observed in the other outcomes. The concept of minimal clinically important difference (MCID) is worth consideration in evaluating the results of this trial. An MCID value of $10 \%$ was found for chronic low back pain Oswestry score and a VAS scale of $20 \mathrm{~mm}$ was found for back pain [15]. In our trial, both MCID values were achieved by the Infliximab group. However, MCID values are known to vary according to the location (local vs radiating) as well as the baseline level of pain [16]. In the case of leg pain, an MCID of c. 33\% reduction in Oswestry score was found to be important regardless of the score level at baseline. In our trial, $50 \%$ reduction in Oswestry was observed, exceeding the value of the Danish trial [16]. Similarly, the treatment differences seen in our trial in RAND-36 physical functioning and bodily pain subscales exceeded the MCID values for these outcomes (7 and 2, respectively) observed in the Danish trial [16]. 
Table 1. Clinical Outcome at Baseline and Week 26 After Single Infusion of Study Agent

\begin{tabular}{|c|c|c|c|c|c|}
\hline & & Infliximab & Placebo & All & P-Value \\
\hline \multirow[t]{2}{*}{ Leg pain $(\mathrm{mm})$} & Baseline & $65(20,84)$ & $70(45,80)$ & $69(20,84)$ & 1.00 \\
\hline & 6-month reduction & $47(2,84)$ & $45(-5,62)$ & $46(-5,84)$ & 0.52 \\
\hline \multirow[t]{2}{*}{ Back pain $(\mathrm{mm})$} & Baseline & $70(8,85)$ & $50(0,95)$ & $64(0,95)$ & 0.60 \\
\hline & 6-month reduction & $27(-26,78)$ & $14(-3,71)$ & $15(-26,78)$ & 0.25 \\
\hline \multirow[t]{3}{*}{ Oswestry Disability Index (\%) } & Baseline & $64(42,74)$ & $50(24,80)$ & $62(24,80)$ & 0.23 \\
\hline & Week 26 & $26(0,72)$ & $54(6,66)$ & $42(0,72)$ & 0.52 \\
\hline & 6-month reduction & $38(-8,60)$ & $2(-4,30)$ & $10(-8,60)$ & 0.57 \\
\hline \multirow[t]{3}{*}{ RAND-36 Bodily Pain } & Baseline & $16(0,45)$ & $28(0,69)$ & $22(0,69)$ & 0.046 \\
\hline & Week 26 & $78(10,90)$ & $45(22,100)$ & $61(10,100)$ & 0.75 \\
\hline & 6-month improvement & $33(0,90)$ & $23(12,45)$ & $27(0,90)$ & 0.83 \\
\hline \multirow[t]{3}{*}{ RAND-36 General Health } & Baseline & $65(50,100)$ & $53(40,80)$ & $58(40,100)$ & 0.17 \\
\hline & Week 26 & $84(80,90)$ & $42(30,60)$ & $61(30,90)$ & 0.021 \\
\hline & 6-month improvement & $0(-5,30)$ & $-10(-25,10)$ & $-3(-25,30)$ & 0.07 \\
\hline Treatment failure at 6 months* & 2 & 3 & 5 & 1.00 & \\
\hline
\end{tabular}

Presented data based on 15 patients at baseline and 12 patients at week 26 (Infliximab N=5; Placebo N=7).

Data presented as median (range) or $\mathrm{n}(\%)$.

* Treatment failures: in the Infliximab group, one patient had discectomy and one had caudal epidural; in the placebo group, one had discectomy and two had caudal epidurals.

In conclusion, in our trial MCID values were reached and exceeded in the Infliximab group, although the differences compared with the placebo group did not reach statistical significance due to low statistical power resulting from slow recruitment. This result was not surprising as trial evidence suggests that placebo treatment had as good effect as the active intervention when disc herniation was located at $\mathrm{L} 5 / \mathrm{S} 1$, whereas placebo was inferior to nerve root injection with a combination of steroid and local anaesthetic [17] or surgery [10] at L4/5. The reason may be anatomical because there is less space at the L4/5 level compared to the L5/S1 level. Hopefully, future trials will clarify the role of TNF $\alpha$ antagonists in the treatment of sciatica, especially on the effect of anti-TNF $\alpha$ agents in the treatment of sciatica due to disc herniations at $\mathrm{L} 3 / 4$ or $\mathrm{L} 4 / 5$.

\section{ACKNOWLEDGEMENT}

Funding for this study was provided by Centocor, Inc., Horsham, PA, USA.

\section{REFERENCES}

[1] Igarashi T, Kikuchi S, Shubayev V, Myers RR. Exogenous tumor necrosis factor- alpha mimics nucleus pulposus-induced neuropathology. Molecular, histologic, and behavioral comparisons in rats. Spine 2000; 25: 2975-80.

[2] Myers RR, Campana WM, Shubayev VI. The role of neuroinflammation in neuropathic pain: mechanisms and therapeutic targets. Drug Discov Today 2006; 11: 8-20.
[3] Karppinen J, Korhonen T, Malmivaara A, et al. Tumor necrosis factor-alpha monoclonal antibody, Infliximab, used to manage severe sciatica. Spine 2003; 28: 750-3.

[4] Korhonen T, Karppinen J, Malmivaara A, et al. Efficacy of Infliximab for disc herniation-induced sciatica: one-year follow-up. Spine 2004; 29: 2115-19.

[5] Korhonen T, Karppinen J, Paimela L, et al. The treatment of disc herniation-induced sciatica with Infliximab: results of a randomized, controlled, 3-month follow-up study. Spine 2005; 30: 2742-8.

[6] Korhonen T, Karppinen J, Paimela L, et al. The treatment of disc herniation-induced sciatica with Infliximab. One-year follow-up results of FIRST II, a randomized controlled trial. Spine 2006; 31: 2759-66.

[7] Luijsterburg PA, Verhagen AP, Ostelo RW, van Os TA, Peul WC, Koes BW. Effectiveness of conservative treatments for the lumbosacral radicular syndrome: a systematic review. Eur Spine J 2007; 16: 881-99.

[8] Weber H. Lumbar disc herniation: a controlled, prospective study with ten years of observation. Spine 1983; 8: 131-40.

[9] Weinstein JN, Lurie JD, Tosteson TD, et al. Surgical vs nonoperative treatment for lumbar disk herniation: the Spine Patient Outcomes Research Trial (SPORT) observational cohort JAMA 2006; 296: 2451-9.

[10] Österman H, Seitsalo S, Karppinen J, Malmivaara A. Effectiveness of microdiscectomy for lumbar disc herniation. A randomised controlled trial with two years of follow-up. Spine 2006; 31: 240914.

[11] Peul WC, van Houwelingen HC, van den Hout WB, et al. Surgery versus prolonged conservative treatment for sciatica. N Engl J Med 2007; 356: 2245-56.

[12] Deyo RA, Weinstein JN. Low back pain. N Engl J Med 2001; 344 363-70. 
[13] Autio R, Karppinen J, Niinimäki J, et al. Determinants of spontaneous resorption of intervertebral disc herniations. Spine 2006; 31: 1247-52.

[14] Autio RA, Karppinen J, Niinimäki J, et al. The effect of Infliximab, a monoclonal antibody against $\mathrm{TNF} \alpha$, on disc herniation resorption: a randomized controlled study. Spine 2006; 31: 2641-5.

[15] Hägg O, Fritzell P, Nordwall A. The clinical importance of changes in outcome scores after treatment for chronic low back pain. Eur Spine J 2003; 12: 12-20.
[16] Lauridsen HH, Hartvigsen J, Manniche C, Korsholm L, GrunnetNilsson N. Responsiveness and minimal clinically important difference for pain and disability instruments in low back pain patients. BMC Musculoskelet Disord 2006; 7: 82.

[17] Karppinen J, Ohinmaa A, Malmivaara A, et al. Cost effectiveness of periradicular infiltration for sciatica: subgroup analysis of a randomized controlled trial. Spine 2001; 26: 2587-95.

(C) Karppinen et al.; Licensee Bentham Open.

This is an open access article licensed under the terms of the Creative Commons Attribution Non-Commercial License (http: //creativecommons.org/licenses/by-nc/

3.0/) which permits unrestricted, non-commercial use, distribution and reproduction in any medium, provided the work is properly cited. 